the carbonyl group comes from the fact that the strongest vibration frequencies accompanying the excitation were found to have values lying between 1400 and $1500 \mathrm{~cm}^{-1}$. A consideration of the intensity distribution in the progressions shows that they correspond to the vibration $1647 \mathrm{~cm} .^{-1}$, of the unexcited state $^{3}$. This is the valence frequency of the $\mathrm{C}=\mathrm{O}$ bond. Thus we have both the vibration and the excitation occurring in the carbonyl group.

In addition to the bands reported above, some very diffuse (pre-ionized) bands were found in the region below $1100 \mathrm{~A}$. superimposed on the ionization continuum. These may well be due to the excitation of non-bonding electrons from the $\mathrm{O}-\mathrm{H}$ group. However, it was not possible to make sufficiently accurate measurements on them to justify an analysis.

$$
\begin{gathered}
\text { University College, } \\
\text { Swansea. }
\end{gathered}
$$

W. M. Evans.

Physical Chemistry Laboratory, Cambridge.

${ }^{1}$ Price, W. C., J. Chem. Phys., 3, 256 (1935).

${ }^{2}$ Mulliken, R. S., J. Chem. Phys., 3, 564 (1935).

s Hibben, J. H., Chem. Rev., 18, 1 (1936).

\section{A Sensitive Adaptation of the Spoon Gauge}

THE spoon gauge is probably the most satisfactory and easily constructed instrument available for the accurate measurement of pressure differences when the use of a manometric liquid is not permissible. Its sensitivity, however, is limited to a pointer movement of about one millimetre for each millimetre of mercury pressure, or perhaps a little more with very fragile gauges. We describe below an effective design which shifts the sensitivity several decimal places into the high vacuum range.

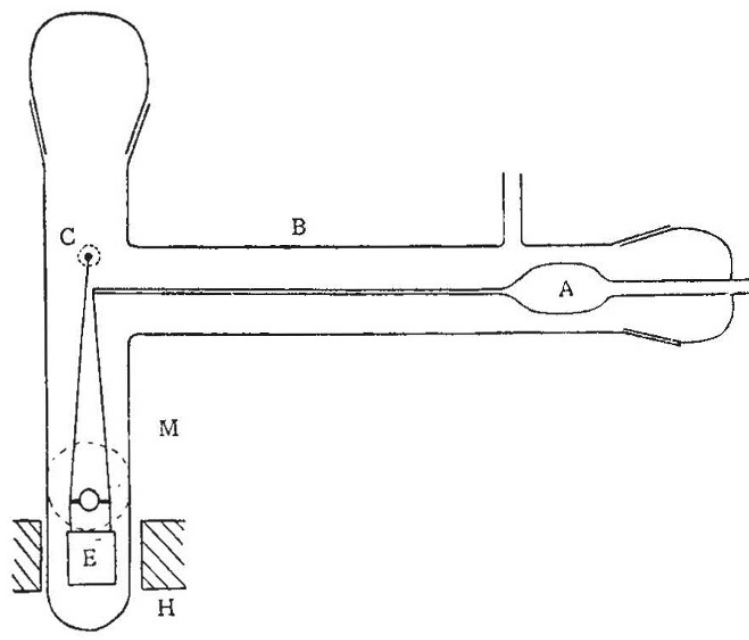

Fig. 1.

The essential feature of the device is a special form of bifilar suspension, which permits amplification of the movement of the pointer end. This suspension consists of unequal and non-parallel threads, the supports of which, placed one above the other, can be brought as closely as desired to the same vertical line. One support is the pointer end, and the other is a movable arm controlling the sensitivity. The threads, spreading out downwards, so as to clear the lower obstructing arm, carry on their ends a horizontal beam to which is attached a mirror and damping device.

The sensitivity depends on the effective horizontal separation of these supports, and this distance can be reduced to less than the thread diameter. Using comparatively rough methods and the usual mirror and scale distances, a magnified movement of about ten thousand-fold is obtainable. With refined methods this figure could be considerably improved.

The particular gauge described below, although simple and convenient in construction, had a sensitivity exceeding one ten thousandth of a millimetre of mercury, and was almost entirely free from vibrational disturbances. A large vertical tube $M$ in Fig. 1, housing the suspension, carries two horizontal tubes at right angles, the lower one $B$ containing the $20 \mathrm{~cm}$. spoon gauge $A$, and the upper one $C$ ending in a ground joint. In this joint could be rotated a slightly eccentric glass arm ending above the tip of the gauge pointer. Long fibres of silk are first attached to the suspension unit, which is lowered into the tube $M$. With each thread touching a support, the beam is adjusted to oscillate axially in the tube about a line parallel with the gauge pointer. The fibres are then sealed to the respective supports with apeison wax and the upper superfluous portions cut short with a hot wire. The sensitivity is raised by rotating sufficiently the cranked rod, and finally all joints are sealed with apeison wax.

The suspension unit consists of a light glass beam, carrying a galvanometer mirror facing a window, and below is fixed a light cylinder of aluminium foil $E$. This rotates in the field of the electromagnet $H$. With critical damping a pressure change is registered with the required accuracy in about two secondsthe natural period of the suspension. The spot light remains stationary within half a millimetre even with the thermostat stirrer running vigorously.

It was found that the zero reading varied somewhat with the magnetic field, due presumably to the magnetic properties of the aluminium vane. For critical damping this amounted to sixty millimetres, and care was taken to maintain a constant field.

This spoon gauge had normally a sensitivity of $8 \mathrm{~mm}$. movement of pointer tip for a pressure change of $10 \mathrm{~mm}$. of mercury. When erected with the scale at two metres from the mirror, the gauge had a sensitivity of $1 \mathrm{~mm}$. deflection for $4 \times 10^{-5} \mathrm{~mm}$. of mercury pressure. This indicated a horizontal separation of the points of suspension of $0.13 \mathrm{~mm}$., which distance is about the minimum that can be easily obtained with the simple arrangement described.

This principle may be used to magnify any horizontal movement.

Alkin Lewis.

D. W. G. Strue.

Chemical Department, King's College, London.

\section{Molecular, Nematic and Crystal States of I : I'- Diethyl- $\psi$-Cyanine Chloride}

Attention has been directed in NATure ${ }^{1}$ to a recent publication by Scheibe, Kandler and Ecker ${ }^{2}$ concerning the narrow absorption and fluorescence band exhibited by certain solutions of the dye $1: 1^{\prime}$-diethyl- $\psi$-cyanine* chloride, in which these authors, on the basis of viscosity measurements,

* This dye is termed 1:1'diethyl- $\psi$-isocyanine in the German publication. 
express the view that the effect is due to a reversible polymerization of ions of the dye. Since the publication of a previous note ${ }^{3}$, in which $I$ have suggested that the narrow absorption band is characteristic of the molecular state, the ageing of aqueous solutions of $1: l^{\prime}$-diethyl- $\psi$-cyanine chloride containing various concentrations of sodium chloride has been studied by the concurrent application of spectrographic, ultramicroscopic and streaming birefringence methods.

Four distinct phases have now been recognized.

lst Phase. Dye in true solution.

2nd Phase. Absorption spectrum changes from that of cationic dye to give a very sharp 'molecular' band at 573-574 $\mathrm{m} \mu$; very strong fluorescence ; uniform luminescence without structure seen with ultra-microscope; no streaming birefringence. This phase is rapidly succeeded by the

3rd Phase. The 'molecular' absorption band at $574 \mathrm{~m} \mu$ shifts towards the red, and becomes less sharp as the preparation ages; fluorescence progressively weakens, and, when excited by plane-polarized light shows partial polarization, whereas the fluorescence similarly excited during the second phase is not polarized; the ultra-microscope shows a fine threadlike structure of a brilliant greenish yellow colour. The threads grow in thickness from about $5 \mathrm{~m} \mu$ to $500 \mathrm{~m} \mu$, while streaming birefringence rapidly in. creases. This phase also shows powerful streaming dichroism.

4th Phase. After some weeks, normal prismatic crystals of the dye are formed.

Crystals of the dye, less than $1 \mu$ thick, exhibit the following trichroism :

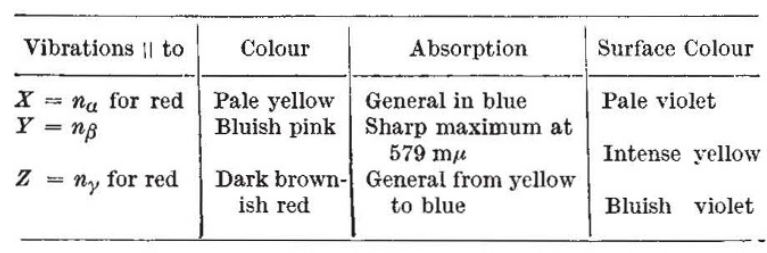

The crystals occasionally form slender needles by elongation along $Y$, and consequently appear bluish pink for vibrations along their length, and any shade from very pale yellow to dark brownish red for vibrations at right angles, according to their orienta. tion. The crystals are not fluorescent.

The optical properties of the threads of the third phase differ in four respects from those of the needle crystals.

(1) The threads are strongly fluorescent, the fluorescence being plane-polarized with vibrations in the length of the thread.

(2) The absorption of light vibrating parallel to the length of the thread at first shows a very sharp maximum at $573 \mathrm{~m} \mu$, which flattens and moves towards the red as the preparation ages, ultimately becoming identical with that of the $Y$ direction of crystals. The threads appear bluish pink for this vibration direction.

(3) The absorption of light vibrating perpendicular to the length is independent of the orientation of the threads, which appear pale orange for such vibrations.

(4) The birefringence is of the same sign for all orientations of the threads, so that the birefringence of threads crossing at a very acute angle is always additive, whereas with random orientations of needle crystals crossing at an acute angle, partial or complete neutralization often occurs.
The threads appear to be true crystals in one dimension $(Y)$ only, for the abnormalities 3 and 4 above are accounted for if we assume that the mole. cules have either free rotation, or else a random orientation, about the $Y$ axis. It is therefore highly probable that the threads of Phase 3 are strictly analogous to the nematic form of 'liquid crystal'. The viscosity abnormalities noted by Scheibe, Kandler and Ecker are therefore explained, not by "polymerization of dye ions" but by the existence of nematic aggregates of dye molecules. The sharp absorption band and associated fluorescence is a characteristic of electronic transitions of individual molecules and their nematic aggregates.

\section{EDWIN E. Jelley.}

Kodak Research Laboratory,

Harrow.

March 11

1 "Research Items", NATURE, 139, 378 (1937).

${ }^{2}$ Scheibe, Kandler and Ecker, Naturwiss., 25, 75 (1937).

3 Jelley, E. E., Nature, 138, 1009 (1936).

\section{Precise Distribution of Mercurialis perennis according to Soil Hydrogen Ion Concentration}

Is a wood covering several acres on the Lower Greensand of Surrey, Mercurialis perennis (dog's mercury) appeared to be distributed in an irregular way, in patches which clearly were not determined by the light intensity alone. In one case it was abundant along the lower side and absent along the upper side of a track that ran across a gentle slope. The pathway had been made up with chalk, and it was clear that the sandy soil below the path had become a possible habitat for Mercurialis because of the washing down of solid calcium carbonate and probably of dissolved bicarbonate as well. The whole region covered by the wood bears scattered patches of chalky soil, owing to transport of chalk for agricultural use and in making up trackways.

Determinations of hydrogen ion concentration were made on soil samples from eight pairs of sites scattered in various parts of the wood. The two sites of each pair were not more than three metres apart (generally within one metre) one being in a patch of Mercurialis and one on ground where it was absent; and each sample was taken from a spot where it was obvious that light was quite adequate but not too strong for the growth of Mercurialis.

The $p \mathrm{H}$ of the eight samples bearing mercury had a mean of $6 \cdot 3$, the values varying between $7 \cdot 3$ and 5.5 ; (free carbonate was present in the only two samples with a $p \mathbf{H}$ above $7 \cdot 0$ ). The $p \mathrm{H}$ of the eight samples from sites with no mercury had a mean of $4 \cdot 8$, the values varying between $5 \cdot 5$ and $4 \cdot 4$.

The absence of Mercurialis perennis from soils with a low quantity of exchangeable calcium has been shown by De Silva ${ }^{1}$ and by Mukerji ${ }^{2}$, and it needs no substantiation. Likewise De Silva has reported the local occurrence of Mercurialis on a spot which had recently been given a higher base status by the dumping of rubbish. What is interesting about the records given here is not so much that the spots bearing Mercurialis have a high $p \mathrm{H}$, as that the similar spots tested where there was no Mercurialis all showed a distinctly lower $p \mathrm{H}$, that is, that Mercurialis has become distributed to all (generalizing from the data collected) the adequately lit spots where the edaphic factors make its growth possible. This is, of course, a state of affairs which one would expect, particularly in a species readily dispersed and 